\title{
Glaciares en el Sistema Nacional de Áreas Silvestres Protegidas por el Estado (SNASPE)
}

\author{
Alexis Segovia Rocha, alexsego@gmail.com
}

\section{RESUMEN}

Se realizó un catastro del número, superficie y equivalente en agua de los glaciares (incluidos los glaciares rocosos) insertos dentro de las categorías operativas del Sistema Nacional de Áreas Silvestres Protegidas por el Estado (SNASPE) a nivel nacional. La determinación del número y la superficie de glaciares dentro de áreas protegidas se realizó mediante el cruce de las bases de datos del inventario nacional de glaciares de la Dirección General de Aguas (DGA), y los polígonos del SNASPE del Ministerio del Medio Ambiente (MMA). Se estimó el equivalente en agua de los glaciares y se asumió una densidad del hielo de $0,9 \mathrm{gr} \mathrm{cm}^{-3}$. Los resultados arrojaron que a nivel nacional el SNASPE tiene bajo su jurisdicción el $43 \%$ de los glaciares y el $83 \%$ de la superficie de hielo del país. No obstante lo anterior, existen grandes diferencias macrozonales puesto que la zona norte y sobre todo la zona centro pueden estar subrepresentadas en términos de conservación glaciológica, con solo el $10 \%$ y el 3,8\% respectivamente de superficie de hielo bajo alguna categoría SNASPE, asunto de gran relevancia, toda vez que la zona norte presenta conflictos permanentes de escasez de agua por su condición de aridez y por competencia entre actividades productivas. La zona centro concentra aproximadamente el 65,9\% de la población a nivel nacional con las consiguientes demandas hídricas que ello implica. Por el contrario, la zona sur $(47 \%)$ y la zona austral $(90 \%)$, presentan gran cantidad de su superficie de hielo bajo alguna categoría SNASPE.

Palabras clave: Chile; glaciar; SNASPE; área silvestre protegida.

\section{Glaciers into the National System of Protected Wild Areas by the State (SNASPE)}

\begin{abstract}
A nationwide register of the quantity, surface and water equivalent of glaciers (including rock glaciers) inserts within the operational categories of the National System of Protected Wild Areas by the State (SNASPE) was conducted. The determination of the number and surface of glaciers within the protected areas was performed by crossing data from the National Inventory of Glaciers of the Water Directorate (DGA) with polygons from SNASPE available on the Ministry of Environment (MMA). The water equivalent of glaciers was estimated and a density of $0,9 \mathrm{gr} \mathrm{cm}^{-3}$ was assumed. The results showed that nationwide SNASPE has under its jurisdiction $43 \%$ of glaciers and $83 \%$ of the ice surface of the country. Nevertheless, there are major macrozone differences since the North with $10 \%$ and especially the Central Zone with only $3.8 \%$ of ice surface under some SNASPE seems to be underrepresented in terms of glaciological conservation. This matter is of great importance, since the Northern Zone has permanent water shortages conflicts because of its arid condition and competition between productive activities. For its part, the Central Zone concentrates approximately $65.9 \%$ of the nationwide population with consequent water demands that implies. On the contrary, the south $(47 \%)$ and the southern region $(90 \%)$ have lots of ice surface under some SNASPE category.
\end{abstract}

Keywords: Chile; glacier; SNASPE; protected wilderness areas.

Recibido el 8 de enero de 2015, aceptado el 1 de julio de 2015 


\section{INTRODUCCIÓN}

Los glaciares juegan un rol esencial en la dinámica natural, especialmente en la continuidad del escurrimiento de los cursos de agua, por lo tanto también influyen en los ciclos ecológicos de los ecosistemas bajo su influencia, además de representar un bienestar general para la población, dado que estos cuerpos de hielo ofrecen variados servicios ecosistémicos tanto de provisión, de regulación y culturales, los cuales toman cada vez más relevancia debido a la merma ambiental acelerada que los glaciares han sufrido en las últimas décadas, presumiblemente debido a los cambios atmosféricos, como el aumento de las temperaturas y la baja en las precipitaciones.

Chile es un país meridional, cuyo principal aporte hídrico en gran parte del territorio es basado en cuencas con dominio nivoglaciar, a su vez, el $63,8 \%\left(477.671 \mathrm{~km}^{2}\right)$ del territorio continental corresponde a zonas de montaña (FAO 2012), lo que hace de Chile un país montaño-dependiente en términos de provisión de agua. La relevancia del aporte glaciar llega a tal punto que PEÑA \& NAZARALA (1987) estimaron que en las cuencas de Chile central representaron alrededor del $67 \%$ del caudal medio mensual del año hidrológico 1968-1969 en la cuenca del Río Maipo.

En Chile al año 2015, los glaciares no tienen un reglamento jurídico y no gozan de protección específica, siendo casi la única forma de resguardo el estar insertos dentro de los límites de algún sitio protegido, debido a que los planes de manejo hacen mención a la zonificación y a las normas de las áreas englaciadas dentro de las zonas protegidas. Debido a esto se hace necesario contar con información que determine la cantidad de glaciares y la superficie de hielo que se encuentra dentro de las distintas categorías de sitios protegidos. En este sentido, el contar con glaciares dentro del Sistema Nacional de Áreas Silvestres
Protegidas por el Estado (SNASPE), se constituye en una cierta garantía para la conservación de estos cuerpos de hielo.

\section{Marco Normativo de Glaciares. Normativa Chilena en torno a los Glaciares}

En la actualidad los glaciares no poseen un estatuto jurídico y tampoco existe una definición legal de glaciar. La protección más explícita de los glaciares es a través del sistema de áreas protegidas, esto debido a que los planes de manejo como instrumentos de gestión hacen mención a la zonificación y a las normas de las áreas englaciadas dentro de dichas zonas. La Ley $\mathrm{N}^{\circ} 20.417$ (que modifica la Ley $\mathrm{N}^{\circ} 19.300$ sobre Bases Generales del Medio Ambiente), estipula que los glaciares situados al interior de las áreas protegidas forman parte de éstas y siguen su régimen. El artículo 10, letra $\mathrm{p}$, de la Ley $\mathrm{N}^{\circ} 19.300$, estipula que las obras, programas o actividades en parques nacionales, reservas nacionales, monumentos naturales, reservas de zonas vírgenes, santuarios de la naturaleza, parques marinos, reservas marinas o en cualesquiera otra área bajo protección oficial, en los casos en que la legislación respectiva lo permita, deberán someterse al sistema de evaluación de impacto ambiental si Los proyectos o actividades son susceptibles de causar impacto ambiental.

La Estrategia Nacional de Glaciares del año 2009, plantea una definición operativa de glaciar, toda vez que es necesario emplear una terminología que permita incluir, desestimar y clasificar los cuerpos de hielo presentes en el territorio nacional, por lo que bajo este punto de vista se establece que glaciar se define como la superficie de hielo y nieve permanente generada sobre suelo, que sea visible por períodos de al menos 2 años y de un área igual o superior a 0,01 $\mathrm{km}^{2}$ (una hectárea). O cualquier superficie rocosa con evidencia superficial de flujo viscoso, producto de un alto contenido de hielo actual o pasado en el subsuelo (DIRECCIÓN GNERAL DE AGUAS- 
CENTRO DE ESTUDIOS CIÉÍTIFICOSs 2009). Esta última es la definición que se utilizó para realizar el inventario nacional de glaciares, por lo tanto, para este estudio se utilizará esta definición de glaciar y sus categorías de clasificación (Glaciarete, Glaciar de Montaña, Glaciar de Valle, Glaciar Efluente y Glaciar Rocoso).

\section{Servicios ecosistémicos de glaciares dentro de áreas protegidas}

El concepto de servicios ecosistémicos se entendiende como aquellos beneficios que las personas obtienen de los ecosistemas, que incluyen servicios de provisión como comida, agua y madera; servicios de regulación tales como regulación de ciclos climáticos, regulación de inundaciones y calidad de aguas; y servicios culturales de tipo recreacional, estéticos y espirituales (Millenium Ecosystem Assessment 2005). En este sentido, gran número de glaciares presentan un potencial turístico desde el punto de vista del disfrute de percepción paisajística por sus atributos morfológicos de carácter estético (tamaño, forma, color, entre otros). Es así como los glaciares sustentan parte de la industria del turismo con actividades del denominado turismo aventura o de intereses especiales, como escalada en hielo, caminatas, travesías, ski, fotografía, avistamiento de caída de témpanos, navegación por fiordos y lagunas proglaciares, entre otros (SEGOVIA 2014). La majestuosidad de estos cuerpos de hielo ofrece un buen complemento para varias zonas que agrupan distintos intereses turísticos, como es el caso de gran cantidad de parques nacionales y reservas naturales, en donde los glaciares son un elemento más de las riquezas turístico-ambientales. Además, la gran importancia de los glaciares como indicadores de cambio climático, sumado a su tendencia mundial de retroceso los hace cada vez más cotizados para conocerlos. También, los glaciares brindan importantes espacios naturales y nutren de agua a diferentes ecosistemas aguas abajo en las áreas silvestres protegidas.

En cuanto a la cuantificación de los beneficios ecosistémicos de los glaciares, en 2014 se realizó una valoración económica de tres servicios ecosistémicos de los glaciares Monumento Natural El Morado (Región Metropolitana), estos servicios fueron Almacenaje de Agua, provisión de Flujo Hídrico Continuo y Turismo y Recreación, para lo cual se estimó que utilizando métodos conservadores de valoración ambiental, los glaciares del Monumento Natural El Morado representan un benéfico económico anual cercano a los 4 mil millones de pesos, lo que es superior en aproximadamente 173 veces el presupuesto total para todo el Monumento Natural El Morado para el año 2013 (SEGOVIA 2014).

\section{Áreas Silvestres Protegidas y Glaciares}

En Chile la legislación contempla una definición de área protegida en el Reglamento del Sistema de Evaluación de Impacto Ambiental, Decreto Supremo $N^{\circ} 95$ de 2001, del Ministerio Secretaría General de la Presidencia como: cualquier porción del territorio, delimitada geográficamente y establecida mediante acto de autoridad pública, colocada bajo protección oficial con la finalidad de asegurar la diversidad biológica, tutelar la preservación de la naturaleza y conservar el patrimonio ambiental.

En 1984 se dictó la ley 18.362, que crearía el Sistema Nacional de Áreas Silvestres Protegidas del Estado (SNASPE), y pese a que nunca ha entrado en vigencia, se encuentra operativo como práctica consuetudinaria para la gestión y administración del patrimonio natural de Chile, y en este sentido, también para la gestión y conservación de ecosistemas de áreas englaciadas. 


\section{Sistema Nacional de Áreas Silvestres Protegidas por el Estado (SNASPE)}

El Sistema Nacional de Áreas Silvestres Protegidas por el Estado (SNASPE), corresponde a aquellos ambientes naturales, terrestres o acuáticos que el Estado protege y maneja para lograr su conservación. El sistema está formado por tres categorías operativas de manejo (Parques Nacionales, Reservas Nacionales y Monumentos Naturales), que fueron analizadas en este estudio para determinar el número y la superficie de glaciares que se encuentra bajo su tutela.

En la actualidad el SNASPE tiene 100 unidades, distribuidas en 36 Parques Nacionales, 49 Reservas Nacionales y 15 Monumentos Naturales, todas ellas administradas por CORPORACIÓN NACIONAL FORESTAL (CONAF):

a) Parque Nacional: Área generalmente extensa, donde existen diversos ambientes únicos o representativos de la diversidad ecológica natural del país, no alterados significativamente por la acción humana, capaces de auto perpetuarse.

b) Reserva Nacional: Área de conservación y protección del recurso suelo y de las especies amenazadas de fauna y flora silvestres, la mantención o mejoramiento de la producción hídrica, y el desarrollo y aplicación de tecnologías de aprovechamiento racional de la flora y la fauna.

c) Monumento Natural: Área destinada a la preservación de muestras de ambientes naturales y de rasgos culturales y escénicos asociados a ellos, y, en la medida que sea compatible con esto, la realización de actividades de educación, investigación y/o recreación.

\section{MATERIALES Y MÉTODOS}

Se utilizó la información provista por el inventario nacional de glaciares de la Dirección General de Aguas (DIRECCIÓN GENERAL DE AGUAS 2014), con en formato Shape para ser trabajado en un software de Sistema de Información Geográfico (SIG). Además se trabajó con la información de los sitios protegidos obtenidos del sistema de información territorial del Ministerio del Medio Ambiente (MMA), con actualización al año 2011, también en formato Shape.

En base al cruce de información entre ambos inventarios (Inventario de Glaciares e Inventario de Sitios Protegidos), y una posterior corrección manual sitio a sitio, se logró establecer la cantidad y superficie de glaciares que se encuentra dentro de alguna categoría de área protegida SNASPE.

Para el cálculo del equivalente en agua de los glaciares, se estimó el espesor medido de cada cuerpo de hielo, para esto se utilizó la fórmula de CHEN \& OHMURA (1990), la cual basa su estimación del espesor relacionando la superficie del glaciar. Este método es un derivado estadístico validado en la observación de 67 glaciares de los Alpes, medidos con técnicas de radar de penetración o sondeos sísmicos:

$$
\text { Espesor }(\mathrm{m})=28,5\left(\text { superficie } \mathrm{km}^{2}\right)^{0,357}
$$

Luego de calcular el espesor medio de cada glaciar, se multiplicó por la superficie para obtener el volumen, para luego aplicar el factor de $0,9 \mathrm{gr} \mathrm{cm}^{-3}$ definida para la densidad del hielo en PATERSON (1994).

Para los glaciares rocosos el tratamiento fue levemente diferente, en el sentido de que primero a su volumen total se le aplicó un factor de 0,5 , equivalente al porcentaje 
de hielo que se estima contienen los glaciares rocosos según BARSCH (1996), para luego aplicar el factor de densidad del hielo de $0,9 \mathrm{gr} \mathrm{cm}^{-3}$.

De esta manera, el cálculo para la estimación del equivalente en agua se traduce de la siguiente manera:

Glaciares descubiertos:

Eq agua $\left(\mathrm{km}^{3}\right)=\left(\right.$ Área $_{\mathrm{km}}^{2} \times$ Espesor $\left._{\mathrm{km}}\right) \times 0,9$

Glaciares rocosos:

$$
\begin{gathered}
\text { Eq agua }\left(\mathrm{km}^{3}\right)=\left[\left(\text { Área }_{\mathrm{km}}{ }^{2} \times \text { Espesor }_{\mathrm{km}}\right) \times\right. \\
0,5] \times 0,9
\end{gathered}
$$

\section{RESULTADOS}

\section{Glaciares dentro el Sistema Nacional de Áreas Silvestres Protegidas por el Estado (SNASPE)}

El SNASPE posee un total de 36 Parques Nacionales $\left(91.403 \mathrm{~km}^{2}\right), 49$ Reservas Nacionales $\left(54.291 \mathrm{~km}^{2}\right)$ y 15 Monumentos Naturales $\left(268 \mathrm{~km}^{2}\right)$, con un total aproximado de $145.963 \mathrm{~km}^{2}$ de superficie.

Chile, según el inventario nacional de glaciares de la Dirección General de Aguas, posee aproximadamente 24.114 glaciares con una superficie de 23.641,4 $\mathrm{km}^{2}$. Estos cuerpos de hielo son relevantes en un amplio abanico de ámbitos, puesto que proveen importantes servicios ecosistémicos como el almacenaje de agua, la regulación y la provisión hídrica de las cuencas, además del disfrute paisajístico como forma de turismo y recreación, entre otros. En este sentido, como los glaciares forman parte de las cabeceras de los distintos ecosistemas desde el norte hasta la zona austral de Chile, es relevante que también se encuentren representados y contenidos dentro de las distintas figuras de protección de áreas silvestres.

\section{Glaciares dentro de Parques Nacionales}

En Chile, la categoría de Parque Nacional representa la mayor figura de protección hacia los glaciares, puesto que además de estar sujeta a las disposiciones de la ley 20.417, está afecta a lo estipulado en el artículo III de la Convención de Washington", donde se estipula que "Los Gobiernos Contratantes convienen en que los límites de los parques nacionales no serán alterados ni enajenada parte alguna de ellos sino por acción de la autoridad legislativa competente. Las riquezas existentes en ellos no se explotarán con fines comerciales".

De los 36 Parques Nacionales presentes en el territorio nacional, 16 presentan glaciares dentro de sus límites. La superficie de hielo dentro de los 16 parques mencionados llega a $18.767,5 \mathrm{~km}^{2}$, lo que representa el $21,3 \%$ del total de superficie de los 16 Parques $\left(87.952,5 \mathrm{~km}^{2}\right)$. Además, seis de estos 16 Parques Nacionales comparten parte de su territorio con la categoría de Reserva de la Biósfera, tal como Lauca, Laguna del Laja, Conguillio, Laguna San Rafael, Alberto de Agostini $^{2}$ y Torres del Paine.

Los Parques Nacionales que poseen más número de glaciares y superficie de hielo son los que se ubican en las regiones de Aysén y Magallanes, dentro de los cuales ordenados de mayor a menor área glaciar son: el Parque Nacional Bernardo O'Higgins con $9.817,6 \mathrm{~km}^{2}$, Laguna San Rafael con 4.615,4 km², Alberto de Agostini con $2.831 .7 \mathrm{~km}^{2}$ y Torres del Paine con 787 $\mathrm{km}^{2}$ (Tabla 1).

1 Convención para la Protección de la Flora, Fauna y las Bellezas Escénicas Naturales de América (1940), y ratificado por Chile en 1967.

$29.668 \mathrm{~km} 2$ de hielo, 43 glaciares del Parque Nacional Alberto de Agostini no son reserva de la Biósfera. 
Tabla 1. Número y superficie (KM²) de glaciares dentro de Parques Nacionales.

Table 1. Number and surface (KM²) of glaciers within National Parks.

\begin{tabular}{|c|c|c|c|c|c|c|}
\hline \multicolumn{7}{|c|}{ CATEGORIA SNASPE: PARQUES NACIONALES } \\
\hline $\mathbf{N}^{\circ}$ & Nombre Unidad & Región & $\begin{array}{c}\text { Superficie Uni- } \\
\text { dad SNASPE } \\
\left(\mathbf{k m}^{2}\right)\end{array}$ & $\begin{array}{c}\mathrm{N}^{\circ} \text { de } \\
\text { glaciares }\end{array}$ & $\begin{array}{c}\text { Superficie } \\
\text { de glaciares } \\
\quad(\mathbf{k m} 2)\end{array}$ & $\begin{array}{c}\text { Porcentaje superficie } \\
\text { glaciar respecto de la } \\
\text { unidad SNASPE }\end{array}$ \\
\hline 1 & *P.N. Lauca & $\begin{array}{c}\text { Arica y } \\
\text { Parinacota }\end{array}$ & $1.372,5$ & 104 & 13,0 & $0,9 \%$ \\
\hline 2 & P.N. Volcán Isluga & Tarapacá & $1.662,3$ & 13 & 0,8 & $0,1 \%$ \\
\hline 3 & P.N. Llullaillaco & Antofagasta & $2.679,2$ & 7 & 0,9 & $0,0 \%$ \\
\hline 4 & *P.N. Laguna del Laja & Biobío & 120,6 & 16 & 7,7 & $6,4 \%$ \\
\hline 5 & *P.N. Conguillio & Araucanía & 606,4 & 56 & 24,0 & $4,0 \%$ \\
\hline 6 & P.N. Villarrica & Araucanía & 600,0 & 38 & 36,7 & $6,1 \%$ \\
\hline 7 & P.N. Corcovado & Los Lagos & $2.928,0$ & 509 & 243,3 & $8,3 \%$ \\
\hline 8 & P.N. Hornopirén & Los Lagos & 483,6 & 196 & 88,4 & $18,3 \%$ \\
\hline 9 & P.N. Puyehue & Los Lagos & $1.131,1$ & 20 & 2,4 & $0,2 \%$ \\
\hline 10 & $\begin{array}{l}\text { P.N. Vicente Perez } \\
\text { Rosales }\end{array}$ & Los Lagos & $2.494,0$ & 47 & 50,5 & $2,0 \%$ \\
\hline 11 & P.N. Isla Magdalena & Aysén & $1.568,4$ & 10 & 3,4 & $0,2 \%$ \\
\hline 12 & $\begin{array}{l}\text { *P.N. Laguna San } \\
\text { Rafael }\end{array}$ & Aysén & $17.107,4$ & 1.884 & $4.615,4$ & $27,0 \%$ \\
\hline 13 & P.N. Queulat & Aysén & $1.572,2$ & 342 & 244,8 & $15,6 \%$ \\
\hline 14 & $\begin{array}{l}\text { P.N. Bernardo } \\
\text { O'Higgins }\end{array}$ & $\begin{array}{c}\text { Aysén - } \\
\text { Magallanes }\end{array}$ & $36.738,4$ & 3.141 & $9.817,6$ & $26,7 \%$ \\
\hline 15 & *P.N. Alberto Agostini & Magallanes & $14.603,9$ & 1.306 & $2.831,7$ & $19,4 \%$ \\
\hline 16 & ${ }^{*}$ P.N. Torres del Paine & Magallanes & $2.284,5$ & 269 & 787,0 & $34,4 \%$ \\
\hline & \multicolumn{2}{|l|}{ Total } & $87.952,5$ & 7.958 & $18.767,5$ & $21,3 \%$ \\
\hline
\end{tabular}

* Además tienen la categoría de Reserva de la Biósfera.

Tabla 2. Número y superficie (KM²) de glaciares dentro de Parques Nacionales, por zonas GLACIOLÓGICAS.

Table 2. Number and surface $\left(\mathrm{KM}^{2}\right)$ of glaciers within National Parks, by glaciological zone.

\begin{tabular}{|c|c|c|c|c|c|c|}
\hline $\begin{array}{c}\text { Zona Glacio- } \\
\text { lógica }\end{array}$ & $\begin{array}{c}\mathbf{N}^{\circ} \text { de } \\
\text { glaciares }\end{array}$ & $\begin{array}{c}\text { Superficie } \\
\text { glaciar } \mathbf{( k m}^{2} \text { ) }\end{array}$ & $\begin{array}{c}\mathbf{N}^{\circ} \mathbf{d e} \\
\text { glaciares } \\
\text { en Parques } \\
\text { Nacionales }\end{array}$ & $\begin{array}{c}\left.\text { Superficie } \mathbf{k m}^{2}\right) \\
\text { glaciar en Par- } \\
\text { ques Nacionales }\end{array}$ & $\begin{array}{c}\% \text { de glaciares } \\
\text { en Parques } \\
\text { Nacionales }\end{array}$ & $\begin{array}{c}\% \text { de Superficie } \\
\text { de glaciares en } \\
\text { Parques Nacio- } \\
\text { nales }\end{array}$ \\
\hline Zona Norte & 2.142 & 180.2 & 124 & 14.7 & $5.8 \%$ & $8.2 \%$ \\
\hline Zona Centro & 2.615 & 854.7 & 0 & 0 & $0 \%$ & $0 \%$ \\
\hline Zona Sur & 5.660 & 1700.8 & 1234 & 701.1 & $21.8 \%$ & $41.2 \%$ \\
\hline Zona Austral & 13.697 & 20905.8 & 6600 & 18051.7 & $48.2 \%$ & $86.3 \%$ \\
\hline Total & $\mathbf{2 4 . 1 1 4}$ & $\mathbf{2 3 6 4 1 . 4}$ & $\mathbf{7 9 5 8}$ & $\mathbf{1 8 7 6 7 . 5}$ & $\mathbf{3 3 \%}$ & $\mathbf{7 9 . 4 \%}$ \\
\hline
\end{tabular}


Las cifras números anteriores, llevados a nivel regional son aún más preocupantes, puesto que como se puede ver en la siguiente tabla, hay una faja que comprende 6 regiones (desde la Región de Atacama hasta la Región del Maule), en que la superficie de glaciares dentro de parques nacionales es inexistente, no obstante lo anterior, dichas 6 regiones en conjunto suman un total de 990 km2 de hielo (Tabla 3 y Figura 1).

Tabla 3. Número y superficie (KM²) de glaciares dentro de Parques Nacionales, por zonas GLACIOLÓGICAS.

Table 3. Number and surface (KM²) of glaciers within National Parks, by glaciological zone.

\begin{tabular}{|c|c|c|c|c|c|c|c|}
\hline $\begin{array}{l}\text { Cod. } \\
\text { Región }\end{array}$ & $\begin{array}{l}\text { Nombre } \\
\text { Región }\end{array}$ & $\begin{array}{l}\mathbf{N}^{\circ} \text { glaciares } \\
\text { en Parques } \\
\text { Nacionales }\end{array}$ & $\begin{array}{c}\text { Superficie } \\
\left(\mathbf{k m}^{2}\right) \text { de } \\
\text { glaciares } \\
\text { en Parques } \\
\text { Nacionales }\end{array}$ & $\begin{array}{l}\mathrm{N}^{\circ} \text { total de } \\
\text { glaciares }\end{array}$ & $\begin{array}{l}\text { Sup. }\left(\mathrm{km}^{2}\right) \\
\text { total de } \\
\text { glaciares }\end{array}$ & $\begin{array}{c}\% \text { de } \\
\text { glaciares } \\
\text { en Parques } \\
\text { nacionales }\end{array}$ & $\begin{array}{l}\% \text { de super- } \\
\text { ficie glaciar } \\
\text { en Parques } \\
\text { Nacionales }\end{array}$ \\
\hline 15 & $\begin{array}{c}\text { Arica y } \\
\text { Parinacota }\end{array}$ & 104 & 13.0 & 327 & 30.4 & $31.8 \%$ & $42.8 \%$ \\
\hline 1 & Tarapacá & 13 & 0.8 & 91 & 6.4 & $14.3 \%$ & $13.1 \%$ \\
\hline 2 & Antofagasta & 7 & 0.9 & 139 & 7.2 & $5.0 \%$ & $12.6 \%$ \\
\hline 3 & Atacama & 0 & 0 & 749 & 87.9 & $0 \%$ & $0 \%$ \\
\hline 4 & Coquimbo & 0 & 0 & 836 & 48.3 & $0 \%$ & $0 \%$ \\
\hline 5 & Valparaíso & 0 & 0 & 715 & 135.8 & $0 \%$ & $0 \%$ \\
\hline 13 & Metropolitana & 0 & 0 & 999 & 388.3 & $0 \%$ & $0 \%$ \\
\hline 6 & O'Higgins & 0 & 0 & 683 & 292.3 & $0 \%$ & $0 \%$ \\
\hline 7 & Maule & 0 & 0 & 218 & 38.2 & $0 \%$ & $0 \%$ \\
\hline 8 & Biobío & 16 & 7.7 & 194 & 39.8 & $8.2 \%$ & $19.3 \%$ \\
\hline 9 & Araucanía & 92 & 45.6 & 140 & 64.5 & $65.7 \%$ & $70.7 \%$ \\
\hline 14 & Los Ríos & 17 & 17.3 & 50 & 36.8 & $34.0 \%$ & $46.9 \%$ \\
\hline 10 & Los Lagos & 756 & 382.3 & 3,225 & 928.9 & $23.4 \%$ & $41.2 \%$ \\
\hline 11 & Aisén & 2,857 & $8,169.4$ & 8,943 & 10214.7 & $31.9 \%$ & $80.0 \%$ \\
\hline \multirow[t]{2}{*}{12} & Magallanes & 4,096 & $10,130.5$ & 6,805 & 11321.9 & $60.2 \%$ & $89.5 \%$ \\
\hline & Total & 7,958 & $18,767.5$ & 24,114 & 23641.4 & $33.0 \%$ & $79.4 \%$ \\
\hline
\end{tabular}




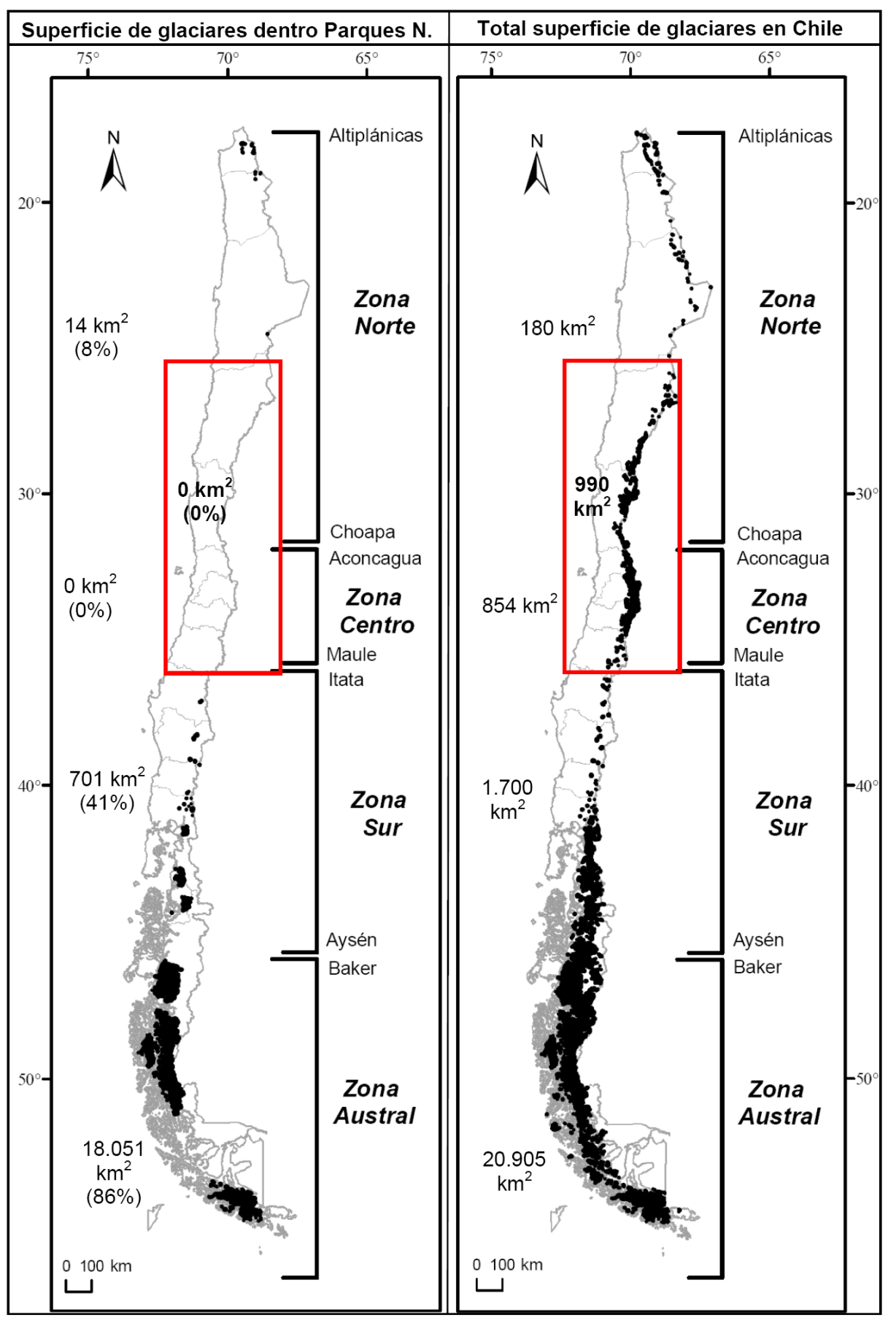

Fugura 1. Número y superficie $\left(\mathrm{km}^{2}\right)$ de glaciares dentro de Parques Nacionales, por zonas glaciológicas.

Figure 1. Number and surface $\left(\mathrm{km}^{2}\right)$ of glaciers within National Parks, by glaciological zone. 
Tabla 4. Número y Superficie (KMํ) De Glaciares dentro de Reservas NaCionales.

Table 4. Number and surface $\left(\mathrm{KM}^{2}\right)$ Of Glaciers within National Reserves.

\begin{tabular}{|c|c|c|c|c|c|c|}
\hline \multicolumn{7}{|c|}{ CATEGORIA SNASPE: RESERVAS NACIONALES } \\
\hline $\mathbf{N}^{\circ}$ & Nombre Unidad & Región & $\begin{array}{l}\text { Superficie } \\
\text { Unidad } \\
\text { SNASPE } \\
\left(\mathbf{k m}^{2}\right)\end{array}$ & $\begin{array}{c}\mathbf{N}^{\circ} \text { de } \\
\text { glaciares }\end{array}$ & $\begin{array}{c}\text { Superficie } \\
\text { de glaciares } \\
\quad\left(\mathbf{k m}^{2}\right)\end{array}$ & $\begin{array}{c}\text { Porcentaje superficie } \\
\text { glaciar respecto de la } \\
\text { unidad SNASPE }\end{array}$ \\
\hline 1 & R.N. Las Vicuñas & $\begin{array}{c}\text { Arica y } \\
\text { Parinacota }\end{array}$ & $2.039,1$ & 40 & 4,1 & $0,2 \%$ \\
\hline 2 & R.N. Río Los Cipreses & O'Higgins & 381,5 & 42 & 30,6 & $8,0 \%$ \\
\hline 3 & *R.N. Ñuble & Biobío & 785,8 & 17 & 1,2 & $0,2 \%$ \\
\hline 4 & R.N. Ralco & Biobío & 133,9 & 22 & 9,4 & $7,0 \%$ \\
\hline 5 & R.N. Malalcahuello & Araucanía & 117,5 & 4 & 0,6 & $0,5 \%$ \\
\hline 6 & R.N. Nalcas & Araucanía & 203,1 & 10 & 1,3 & $0,7 \%$ \\
\hline 7 & R.N. Villarrica & Araucanía & 691,5 & 34 & 16,3 & $2,4 \%$ \\
\hline 8 & $\begin{array}{l}\text { R.N. Mocho } \\
\text { Choshuenco }\end{array}$ & Los Ríos & 75,5 & 17 & 18,2 & $24,1 \%$ \\
\hline 9 & R.N. Futaleufú & Los Lagos & 118,8 & 6 & 1,3 & $1,1 \%$ \\
\hline 10 & R.N. Llanquihue & Los Lagos & 339,9 & 10 & 1,7 & $0,5 \%$ \\
\hline 11 & R.N. Cerro Castillo & Aysén & $1.388,8$ & 268 & 61,1 & $4,4 \%$ \\
\hline 12 & R.N. Katalalixar & Aysén & $7.273,4$ & 88 & 29,5 & $0,4 \%$ \\
\hline 13 & R.N. Lago Jeinimeni & Aysén & $1.599,3$ & 636 & 143,3 & $9,0 \%$ \\
\hline 14 & R.N. Lago Palena & Los Lagos & 387,6 & 62 & 9,0 & $2,3 \%$ \\
\hline 15 & R.N. Lago las Torres & Aysén & 170,3 & 17 & 1,5 & $0,9 \%$ \\
\hline 16 & R.N. Lago Rosselot & Aysén & 123,3 & 1 & 0,1 & $0,1 \%$ \\
\hline 17 & R.N. Río Simpson & Aysén & 420,8 & 45 & 4,2 & $1,0 \%$ \\
\hline 18 & R.N. Alacalufes & Magallanes & $22.988,2$ & 1.272 & 629,6 & $2,7 \%$ \\
\hline & \multicolumn{2}{|l|}{ Total } & $39.238,2$ & 2.591 & 963.1 & $2,5 \%$ \\
\hline
\end{tabular}

* Forma parte de una Reserva de la Biósfera.

\section{Glaciares dentro de Reservas Nacionales}

Las Reservas Nacionales representan una forma de protección más baja con respecto a los Parques Nacionales, puesto que no son mencionadas en un artículo especial en la Convención de Washington, por lo que solo están afectas a las disposiciones de la Ley de Bases de Medio Ambiente y a sus modificaciones mediante la ley 20.417, es decir, que los glaciares situados al interior de las áreas protegidas forman parte de éstas y siguen su régimen, como también que las obras, programas o actividades en Parques Nacionales, Reservas Nacionales, Monumentos Naturales, o en cualesquiera otra área bajo protección oficial, en los casos en que la legislación respectiva lo permita, deberán someterse al sistema de evaluación de impacto ambiental si los proyectos o actividades son susceptibles de causar impacto ambiental (Ley 19.300). 
De las 49 Reservas Nacionales que integran el SNASPE (con un total de $54.291 \mathrm{~km}^{2}$ ), 18 presentan glaciares dentro de sus límites. La superficie de hielo dentro de las 18 reservas mencionadas llega a $963,1 \mathrm{~km}^{2}$, lo que representa el 2,5\% del total de superficie de las 18 Reservas $\left(39.238 \mathrm{~km}^{2}\right)$. Para el caso de las Reservas Nacionales, solo una con presencia de glaciares comparte además la categoría de Reserva de la Biósfera, la cual corresponde a la Reserva Nacional Ñuble en la Región del Biobío. Las Reservas Nacionales que poseen más número de glaciares y superficie de hielo son las que se ubican en las regiones de Aysén y Magallanes, dentro de las cuales ordenadas de mayor a menor área glaciar son las siguientes: Reserva Nacional Alacalufes con $629,6 \mathrm{~km}^{2}$; Jeinimeni con 143,3 $\mathrm{km}^{2}$ y Cerro Castillo con 61,1 km² (Tabla 4).

\section{Glaciares dentro de Monumentos Naturales}

Al igual que las Reservas Nacionales, los Monumentos Naturales tampoco cuentan con un artículo especial en la Convención de Washington, por lo que también están afectos solo a las disposiciones de la ley de bases de medio ambiente. De los 15 Monumentos Naturales repartidos en $268 \mathrm{~km}^{2}$ que integran el SNASPE, solo el Monumento Natural El Morado en la Región Metropolitana presenta glaciares dentro de sus límites. Los cuatro glaciares presentes en el Monumento Natural. El Morado suma una superficie de hielo que alcanza los $1,76 \mathrm{~km}^{2}$, lo que representa el $6,2 \%$ del total de superficie del monumento $\left(28,3 \mathrm{~km}^{2}\right)$ (Tabla 5).

Tabla 5. Número y SUPerficie (KM² ${ }^{2}$ de GlaCiares dentro de Monumentos Naturales.

Table 5. Number and SURface $\left(\mathrm{KM}^{2}\right)$ Of Glaciers within Natural Monuments.

\begin{tabular}{|c|c|c|c|c|c|c|}
\hline \multicolumn{7}{|c|}{ CATEGORIA SNASPE: MONUMENTOS NATURALES } \\
\hline $\mathbf{N}^{\circ}$ & Nombre Unidad & Región & $\begin{array}{c}\text { Superficie } \\
\text { Unidad } \\
\text { SNASPE } \\
\left(\mathbf{k m}^{2}\right)\end{array}$ & $\begin{array}{c}\mathbf{N}^{\circ} \text { de } \\
\text { glaciares }\end{array}$ & $\begin{array}{c}\text { Superficie } \\
\text { de glaciares } \\
\left(\mathbf{k m}^{2}\right)\end{array}$ & $\begin{array}{c}\text { Porcentaje superficie } \\
\text { glaciar respecto de la } \\
\text { unidad SNASPE }\end{array}$ \\
\hline 1 & M.N. El Morado & Metropolitana & 28,3 & 4 & 1,7 & $6,2 \%$ \\
\hline
\end{tabular}

Glaciares dentro del SNASPE a Nivel Nacional

A nivel nacional el SNASPE tiene bajo su jurisdicción el 43,8\% del número de glaciares, y el $83,5 \%$ de la superficie de hielo del país (Fig. 2). No obstante lo anterior, y pese a que los números enunciados anteriormente resultan alentadores en términos de asegurar la conservación de glaciares, existen grandes diferencias regionales, presentando regiones sin glaciares dentro de alguna figura del SNASPE, como las de Atacama, Coquimbo, Valparaíso y del Maule. Por el contrario, hay regiones que superan el $80 \%$ de superficie glaciar dentro de alguna figura de conservación, como la Araucanía, Los Ríos, Aysén y Magallanes (Tabla 6). 


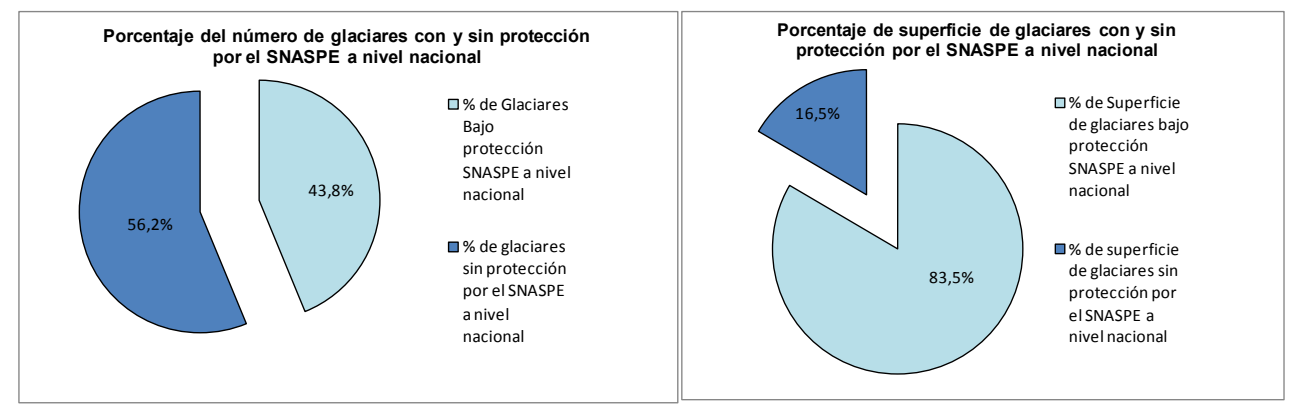

Figura. 2. Porcentaje de glaciares (izquierda) y superficie (derecha) con y sin protección por el SNASPE a nivel nacional.

Figura. 2 Percentage of glaciers (left) and surface (right) with and without protection SNASPE.

TABLA 6. NÚMERo DE GLACIARES Y SUPERFICIE CON PROTECCIÓN POR EL SNASPE A NIVEL NACIONAL.

TAble 6. Number of glaciers and SURFACE Protection SNASPE.

\begin{tabular}{|c|c|c|c|c|c|c|}
\hline Región & $\begin{array}{c}\mathbf{N}^{\circ} \text { de } \\
\text { glaciares } \\
\text { por Región }\end{array}$ & $\begin{array}{c}\text { Superficie } \\
\text { glaciar por } \\
\text { Región } \\
\mathbf{( k m}^{\mathbf{2}}\end{array}$ & $\begin{array}{c}\mathbf{N}^{\circ} \text { de } \\
\text { glaciares bajo } \\
\text { SNASPE }\end{array}$ & $\begin{array}{c}\text { Superficie } \\
\text { glaciar bajo } \\
\text { SNASPE } \\
\mathbf{( k m}^{2} \mathbf{)}\end{array}$ & $\begin{array}{c}\text { \% de } \\
\text { glaciares Bajo } \\
\text { protección } \\
\text { SNASPE }\end{array}$ & $\begin{array}{c}\text { \% de Superficie } \\
\text { de glaciares bajo } \\
\text { SNASPE }\end{array}$ \\
\hline $\begin{array}{c}\text { Arica y } \\
\text { Parinacota }\end{array}$ & 327 & 30,4 & 144 & 17,1 & $44,0 \%$ & $56,2 \%$ \\
\hline Tarapacá & 91 & 6,4 & 13 & 0,8 & $14,3 \%$ & $13,0 \%$ \\
\hline Antofagasta & 139 & 7,2 & 7 & 0,9 & $5,0 \%$ & $12,6 \%$ \\
\hline Atacama & 749 & 87,9 & 0 & 0 & $0 \%$ & $0 \%$ \\
\hline Coquimbo & 836 & 48,3 & 0 & 0 & $0 \%$ & $0 \%$ \\
\hline Valparaíso & 715 & 135,8 & 0 & 0 & $0 \%$ & $0 \%$ \\
\hline Metropolitana & 999 & 388,3 & 4 & 1,8 & $0,4 \%$ & $0,5 \%$ \\
\hline O'Higgins & 683 & 292,3 & 42 & 30,6 & $6,1 \%$ & $10,5 \%$ \\
\hline Maule & 218 & 38,2 & 0 & 0 & $0 \%$ & $0 \%$ \\
\hline Biobío & 194 & 39,8 & 62 & 18,9 & $32,0 \%$ & $47,5 \%$ \\
\hline Araucanía & 140 & 64,5 & 133 & 63,3 & $95,0 \%$ & $98,2 \%$ \\
\hline Los Ríos & 50 & 36,8 & 34 & 35,5 & $68,0 \%$ & $96,6 \%$ \\
\hline Los Lagos & 3.225 & 928,9 & 831 & 391,8 & $25,8 \%$ & $42,2 \%$ \\
\hline Aysén & 8.943 & $10.214,7$ & 3.915 & $8.411,7$ & $43,8 \%$ & $82,3 \%$ \\
\hline Magallanes & 6.805 & $11.321,9$ & 5.368 & $10.760,1$ & $78,9 \%$ & $95,0 \%$ \\
\hline Total & $\mathbf{2 4 . 1 1 4}$ & $\mathbf{2 3 . 6 4 1 , 4}$ & $\mathbf{1 0 . 5 5 3}$ & $\mathbf{1 9 . 7 3 2 , 4}$ & $\mathbf{4 3 , 8} \%$ & $\mathbf{8 3 , 5 \%}$ \\
\hline
\end{tabular}


En un análisis por zona glaciológica, se puede notar que la zona austral (desde la cuenca del río Baker al extremo austral del Chile) tiene el 90,3\% de su superficie glaciar bajo alguna categoría SNASPE, luego sigue la zona sur (Desde la cuenca del Río Itata hasta la cuenca del Río Aysén) con 47,1\%; zona norte (desde el límite con Perú hasta la cuenca del Río Choapa) con $10,4 \%$ y por último la zona centro (Desde la cuenca del río Aconcagua hasta la cuenca del Río Maule) con 3,8\% (Tabla 7 y Fig. 3). Estos datos muestran grandes diferencias macrozonales puesto que la zona norte y sobre todo la zona centro parece estar subrepresentadas en términos de conservación glaciológica, asunto de gran relevancia, toda vez que la zona norte presenta conflictos permanentes de escasez hídrica producto de las características climáticas de baja pluviosidad, la calidad del agua potable y la competencia entre las actividades mineras, industriales y agrícolas. Por su parte, la zona centro concentra casi el $65,9 \%$ de la población a nivel nacional ${ }^{4}, \mathrm{y}$ es la zona de mayor producción agrícola tanto para el abastecimiento interno como de exportación. Además, está presentando desde el año 2012 conflictos con actividades mineras con planes de establecerse y/o ampliarse en la cabecera de algunas cuencas del Aconcagua y con potencial influencia en la cuenca del río Maipo.

TABLA 7. NÚMERO Y SUPERFICIE DE GLACIARES BAJO PROTECCIÓN POR EL SNASPE POR ZONA GLACIOLÓGICA.

TABLe 7. Number aNd SURFACE OF GLACIERS by GLACIOLOGICAL ZONE UNDER PROTECTION SNASPE.

\begin{tabular}{|c|c|c|c|c|c|c|}
\hline $\begin{array}{c}\text { Zona Glacio- } \\
\text { lógica }\end{array}$ & $\begin{array}{c}\mathbf{N}^{\circ} \text { de } \\
\text { glaciares }\end{array}$ & $\begin{array}{c}\text { Superficie } \\
\text { glaciar } \\
\left.\mathbf{( k m}^{2}\right)\end{array}$ & $\begin{array}{c}\mathbf{N}^{\circ} \text { de } \\
\text { glaciares } \\
\text { bajo } \\
\text { SNASPE }\end{array}$ & $\begin{array}{c}\left.\text { Superficie } \mathbf{( k m}^{\mathbf{2}}\right) \\
\text { glaciar bajo } \\
\text { SNASPE }\end{array}$ & $\begin{array}{c}\text { \% de glaciares } \\
\text { Bajo protección } \\
\text { SNASPE }\end{array}$ & $\begin{array}{c}\text { \% de Superficie de } \\
\text { glaciares bajo } \\
\text { protección } \\
\text { SNASPE }\end{array}$ \\
\hline Zona Norte & 2.142 & 180,2 & 164 & 18,8 & $7,7 \%$ & $10,4 \%$ \\
\hline Zona Centro & 2.615 & 854,7 & 46 & 32,4 & $1,8 \%$ & $3,8 \%$ \\
\hline Zona Sur & 5.660 & $1.700,8$ & 1.653 & 800,4 & $29,2 \%$ & $47,1 \%$ \\
\hline Zona Austral & 13.697 & $20.905,8$ & 8.690 & $18.880,9$ & $63,4 \%$ & $90,3 \%$ \\
\hline Total & $\mathbf{2 4 . 1 1 4}$ & $\mathbf{2 3 . 6 4 1 , 4}$ & $\mathbf{1 0 . 5 5 3}$ & $\mathbf{1 9 . 7 3 2 , 4}$ & $\mathbf{4 3 , 8} \%$ & $\mathbf{8 3 , 5} \%$ \\
\hline
\end{tabular}

Dentro de las diferencias totales por tipos morfológicos de glaciares entre las distintas zonas glaciológicas se puede mencionar que en la zona norte se aprecia un fuerte predominio de los glaciares rocosos, los cuales representan el $64 \%$ del número de glaciares y el $51 \%$ de la superficie total de hielo de la zona glaciológica. En importancia de superficie los siguen los glaciares de montaña y luego los glaciaretes. Cabe señalar que no hay presencia de glaciares de valle en la zona norte. Asimismo, en la zona centro la distribución de glaciares según la clasificación morfológica también muestra una preponderancia en el número de glaciares rocosos representando el 55\%, siendo la zona glaciológica que contiene más glaciares de este tipo (1.456). No obstante lo anterior, el mayor aporte en superficie lo constituyen los glaciares de montaña con

4 INE, 2010. Compendio Estadístico, Estadísticas Demográficas. 


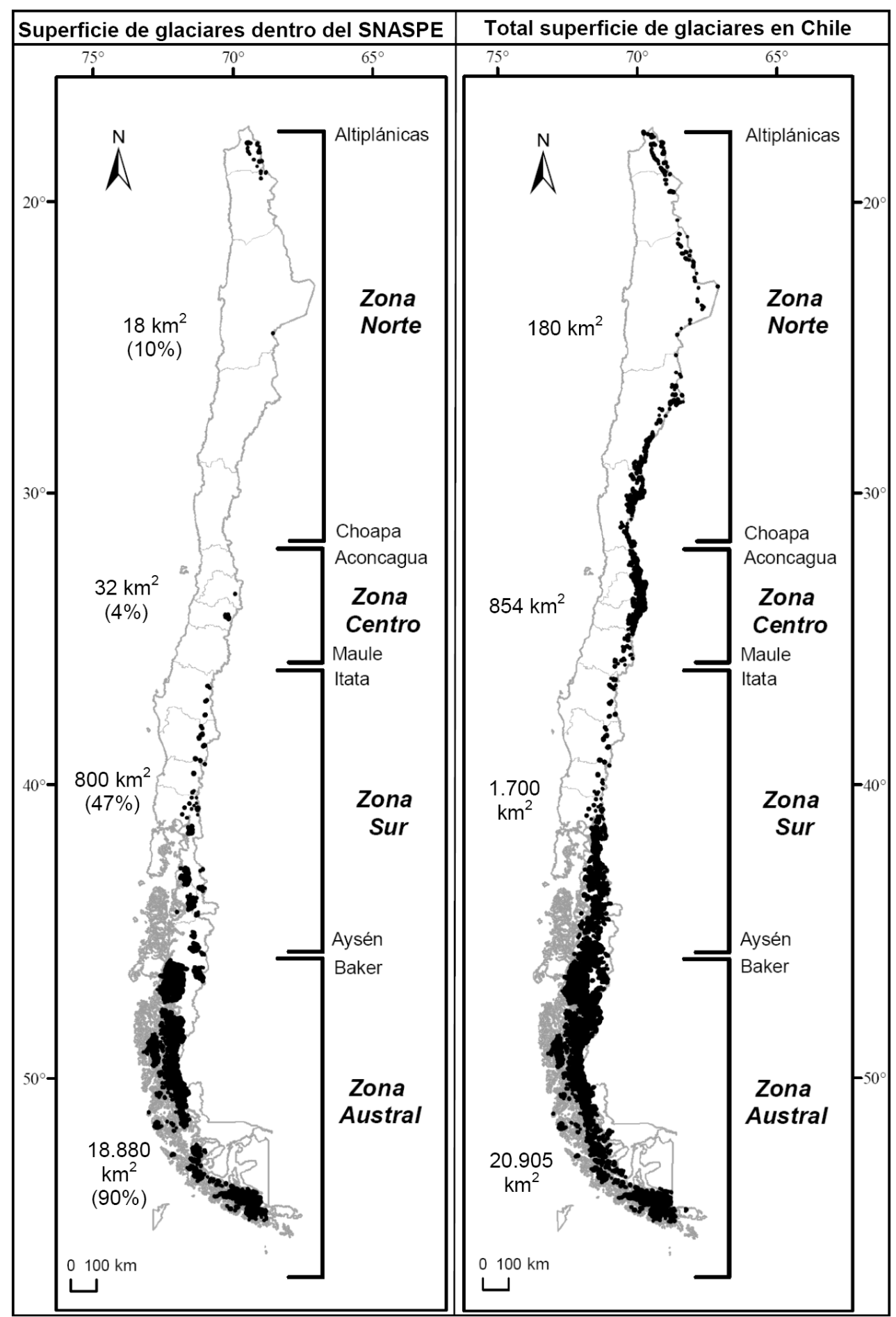

Figura 3. Superficie de glaciares bajo protección por el SNASPE por zona glaciológica.

Figure 3. Surface of glaciers by glaciological zone under protection SNASPE. 
el $33 \%$. En esta zona ya se nota presencia de glaciares de Valle ( 79 glaciares) con el $30 \%$ de la superficie total englaciada de la zona centro. Por el contrario, en la zona sur ya no se detecta la presencia de glaciares rocosos y predominan los glaciares de tipo glaciaretes (3.467) con el 61\%.No obstante lo anterior, el mayor tamaño de los glaciares de montaña hacen que este tipo de cuerpos de hielo aporten el $81 \%$ de la superficie de la zona sur. Por último en la zona austral están los dos grandes campos de hielo (Campo de Hielo Norte y Sur), los cuales representan el $66 \%$ de la superficie de la zona glaciológica y el 58\% del total del hielo nacional, convirtiéndolos por ende, en los mayores cuerpos de hielo del país con $13.851 \mathrm{~km} 2$ de superficie. En cuanto al tipo morfológico de glaciares, dentro del SNASPE por zona glaciológica, estos se aprecian en la tabla 8.

TABLA 8. NúMERo Y SUPERFICIE DE GLACIARES POR TIPO, BAJO PROTECCIÓN POR EL SNASPE POR ZONA GLACIOLÓGICA.

TABLE 8. NUMBER AND SURFACE OF GLACIERS BY TYPE, BY GLACIOLOGICAL ZONE UNDER PROTECTION SNASPE.

\begin{tabular}{|c|c|c|c|c|c|c|c|c|c|}
\hline \multirow[b]{2}{*}{$\begin{array}{l}\text { Clasificación } \\
\text { Morfológica }\end{array}$} & \multirow[b]{2}{*}{$\mathbf{N}^{\circ}$ y Superficie } & \multicolumn{2}{|c|}{ Zona Norte } & \multicolumn{2}{|c|}{ Zona Centro } & \multicolumn{2}{|c|}{ Zona Sur } & \multicolumn{2}{|c|}{ Zona Austral } \\
\hline & & TOTAL & SNASPE & TOTAL & SNASPE & TOTAL & SNASPE & TOTAL & SNASPE \\
\hline \multirow{2}{*}{ Glaciarete } & $\mathrm{N}^{\circ}$ de Glaciares & 579 & 41 & 639 & 24 & 3.467 & 948 & 7.912 & 4.899 \\
\hline & Superficie $\left(\mathrm{km}^{2}\right)$ & 19,7 & 1,7 & 25,7 & 1,1 & 124,3 & 34,3 & 276,1 & 173,7 \\
\hline \multirow{2}{*}{ Glaciar Rocoso } & $\mathrm{N}^{\circ}$ de Glaciares & 1.375 & 86 & 1.456 & 2 & $\mathrm{~s} / \mathrm{r}$ & $\mathrm{s} / \mathrm{r}$ & $\mathrm{s} / \mathrm{r}$ & $\mathrm{s} / \mathrm{r}$ \\
\hline & Superficie $\left(\mathrm{km}^{2}\right)$ & 93,7 & 7,1 & 276,2 & 0,1 & $\mathrm{~s} / \mathrm{r}$ & $\mathrm{s} / \mathrm{r}$ & $\mathrm{s} / \mathrm{r}$ & $\mathrm{s} / \mathrm{r}$ \\
\hline \multirow{2}{*}{$\begin{array}{l}\text { Glaciar de } \\
\text { Montaña }\end{array}$} & $\mathrm{N}^{\circ}$ de Glaciares & 188 & 37 & 441 & 17 & 2.161 & 684 & 5.229 & 3.282 \\
\hline & Superficie $\left(\mathrm{km}^{2}\right)$ & 66,8 & 10 & 288,9 & 11,4 & $1.391,5$ & 643,6 & $4.870,7$ & $3.376,5$ \\
\hline \multirow{2}{*}{ Glaciar de Valle } & $\mathrm{N}^{\circ}$ de Glaciares & $\mathrm{s} / \mathrm{r}$ & $\mathrm{s} / \mathrm{r}$ & 79 & 3 & 32 & 21 & 190 & 143 \\
\hline & Superficie $\left(\mathrm{km}^{2}\right)$ & $\mathrm{s} / \mathrm{r}$ & $\mathrm{s} / \mathrm{r}$ & 263,8 & 19,8 & 185 & 122,6 & $1.907,9$ & $1.479,6$ \\
\hline \multirow{2}{*}{$\begin{array}{l}\text { Glaciar Efluente } \\
\text { de Campo Hielo }\end{array}$} & $\mathrm{N}^{\circ}$ de Glaciares & $\mathrm{s} / \mathrm{r}$ & $\mathrm{s} / \mathrm{r}$ & $\mathrm{s} / \mathrm{r}$ & $\mathrm{s} / \mathrm{r}$ & $\mathrm{s} / \mathrm{r}$ & $\mathrm{s} / \mathrm{r}$ & 366 & 366 \\
\hline & Superficie $\left(\mathrm{km}^{2}\right)$ & $\mathrm{s} / \mathrm{r}$ & $\mathrm{s} / \mathrm{r}$ & $\mathrm{s} / \mathrm{r}$ & $\mathrm{s} / \mathrm{r}$ & $\mathrm{s} / \mathrm{r}$ & $\mathrm{s} / \mathrm{r}$ & 13851.1 & 13851.1 \\
\hline \multirow{2}{*}{$\begin{array}{c}\text { Total por Zona } \\
\text { Glaciológica }\end{array}$} & $\mathrm{N}^{\circ}$ de Glaciares & 2.142 & 164 & 2.615 & 46 & 5.660 & 1.653 & 13.697 & 8.690 \\
\hline & Superficie $\left(\mathrm{km}^{2}\right)$ & 180,2 & 18,8 & 854,7 & 32,4 & $1.700,8$ & 800,4 & $20.905,8$ & $18.880,9$ \\
\hline
\end{tabular}

Tabla 9. Equivalente en agua de los glaciares dentro de alguna categoría SNASPE.

TAble 9. WATER EQuivalent OF GLACIERS IN ALl SNASPE CATEgory.

\begin{tabular}{|c|c|c|c|c|}
\hline CATEGORÍA SNASPE & $\begin{array}{c}\mathbf{N}^{\circ} \text { DE } \\
\text { GLACIARES }\end{array}$ & $\begin{array}{c}\text { SUPERFICIE } \\
\mathbf{k m}^{\mathbf{2}}\end{array}$ & $\begin{array}{c}\text { Eq. AGUA } \\
\mathbf{k m}^{\mathbf{3}}\end{array}$ & $\begin{array}{c}\text { \% Eq. En agua por } \\
\text { categoría SNASPE }\end{array}$ \\
\hline PARQUE NACIONAL & 7958 & 18767,5 & 2999,2 & $98,7 \%$ \\
\hline RESERVA NACIONAL & 2591 & 963,1 & 38,9 & $1,3 \%$ \\
\hline MONUMENTO NATURAL & 4 & 1,8 & 0,04 & $0,001 \%$ \\
\hline TOTAL & $\mathbf{1 0 5 5 3}$ & $\mathbf{1 9 7 3 2 , 4}$ & $\mathbf{3 0 3 8 , 1}$ & $\mathbf{1 0 0} \%$ \\
\hline
\end{tabular}




\section{Equivalente en agua de los glaciares dentro del SNASPE}

En cuanto al equivalente en agua contenida en los glaciares dentro del SNASPE, se puede establecer que el 98\% del agua contenida en los glaciares del SNASPE se encuentra dentro de Parques Nacionales, relegando a un $1,3 \%$ a las Reservas Nacionales y solo a un $0,001 \%$ a los Monumentos Naturales (Tabla 9).

En cuanto a la cantidad equivalente en agua de los glaciares bajo protección del SNASPE, se puede establecer que los glaciares bajo protección SNASPE representan el 95,7\% del equivalente en agua de todos los glaciares del país. No obstante lo anterior, las desigualdades regionales son patentes, por cuanto en la zona norte, el 10,3\% del equivalente en agua de los glaciares se encuentra bajo protección del SNASPE, lo que a modo de comparación, equivale a 0,3 embalses La Paloma $\left(0,75 \mathrm{~km}^{3}\right)$. Para la zona centro, la representatividad del SNASPE llega al 5,5\%, equivalente a 1,9 veces el embalse La Paloma, para la zona sur este número se eleva al $56 \%$ equivalente a 41,3 embalses La Paloma, y para la zona Austral, la representatividad del SNASPE en el equivalente en agua llega a un 97,2\%, equivalente a 4007,3 veces el embalse La Paloma (Tabla 10).

Tabla 10. Equivalente en agua de los glaciares dentro de alguna Categoría SNASPE por ZONA GLACIOLÓGICA.

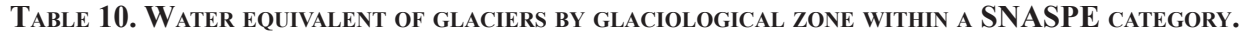

\begin{tabular}{|c|c|c|c|}
\hline ZONA GLACIOLÓGICA & $\begin{array}{c}\text { Equivalente en agua de } \\
\text { todos los glaciares de } \\
\text { Chile } \mathbf{( k m}^{3} \text { ) }\end{array}$ & $\begin{array}{c}\text { Eq, AGUA km } \\
\text { SNASPE }\end{array}$ & $\begin{array}{c}\text { \% Eq agua } \\
\text { SNASPE }\end{array}$ \\
\hline ZONA NORTE & 2,3 & 0,2 & $10,3 \%$ \\
\hline ZONA CENTRO & 26,6 & 1,5 & $5,5 \%$ \\
\hline ZONA SUR & 55,3 & 31,0 & $56,0 \%$ \\
\hline ZONA AUSTRAL & 3091,4 & 3005,5 & $97,2 \%$ \\
\hline TOTAL & $\mathbf{3 1 7 5 , 7}$ & $\mathbf{3 0 3 8 , 1}$ & $\mathbf{9 5 , 7} \%$ \\
\hline
\end{tabular}

\section{CONCLUSIONES}

A nivel nacional, el SNASPE tiene bajo su jurisdicción el 43\% de los glaciares y el $83 \%$ de la superficie de hielo del país. No obstante lo anterior, existen grandes diferencias regionales, existiendo regiones sin glaciares dentro de alguna figura del SNASPE, como las regiones de Atacama, Coquimbo, Valparaíso y del Maule. Por el contrario, hay regiones que superan el $80 \%$ de superficie glaciar dentro de alguna figura de sitio protegido, como la Araucanía, Los Ríos, Aysén y Magallanes. Asimismo, en el análisis por zona glaciológica, también se pueden notar grandes diferencias macrozonales puesto que la zona Norte y sobre todo la zona centro parecieran estar subrepresentadas en términos de conservación glaciológica, asunto de gran relevancia, toda vez que la zona norte presenta conflictos permanentes de escasez de agua producto de la competencia entre las actividades mineras, industriales y agrícolas, y por su parte, la zona centro concentra casi el $65,9 \%$ de la población a nivel nacional, además de estar presentando desde el año 2012 conflictos con actividades mineras con planes de ampliarse en la cabecera de algunas cuencas del Aconcagua y con 
influencia en la cuenca del río Maipo. El escenario anterior se agrava aún más cuando se diferencia solo los glaciares presentes dentro de Parques Nacionales, puesto que se produce una franja sin glaciares en alguna categoría de sitio protegido que abarca seis regiones (desde Atacama hasta el Maule), por lo que la discusión legislativa sobre cuales glaciares quedarán totalmente resguardados para su preservación no debe ser menor, en el sentido de que con solo poner en categoría de preservación los glaciares que se encuentren dentro de Parques Nacionales, priva de los servicios ecosistémicos de los glaciares a seis regiones que actualmente presentan complicaciones de escasez hídrica, además de conflictos por competencia de actividades en zonas englaciadas, por lo que toda esta franja que comprende parte de la zona norte y toda la zona centro quedaría en una incertidumbre acerca la conservación y/o preservación de los glaciares, con los consiguientes problemas de mantención de los servicios ecosistémicos tanto de provisión, regulación y culturales que estos proveen.

En cuanto a la cantidad equivalente en agua de los glaciares bajo protección del SNASPE, se pudo establecer que los glaciares bajo protección SNASPE representan el $95,7 \%$ del equivalente en agua de todos los glaciares del país. No obstante lo anterior, las desigualdades macrozonales son patentes teniendo poca representatividad la zona norte y centro y muy alta representatividad la zona sur y austral.

El poder contar con SNASPE y con otras categorías de áreas protegidas representa un beneficio para la conservación de los glaciares que se encuentran dentro de ellas, y en este sentido, es de suma relevancia propender a una representación regional y zonal eficaz de la cantidad de glaciares protegidos en relación al total, de modo de proteger y conservar la gran variedad de servicios ecosistémicos que estos proveen.

\section{AGRADECIMIENTOS}

A CONICYT, Dirección General de Aguas (DGA); Ministerio del Medio Ambiente (MMA), Corporación Nacional Forestal (CONAF), Facultad de Ciencias Forestales y de la Conservación de la Naturaleza de la Universidad de Chile (FCFCN).

\section{REFERENCIAS}

BARSCH, D., 1996. Rockglaciers. Indicators for the Present and Former Geoecology in High Mountain Environments. Springer Verlag, Heidelberg. $331 p$.

CHEN, J. \& A. OHMURA, 1990. Estimation of Alpine glacier water resources and their change since the 1870s. In Hydrology in Mountainous Regions, I - Hydrological Measurements; the Water Cycle, Proceedings of two Lausanne Symposia, August 1990, Lang H, Musy A (eds). 10p.

\section{DIRECCION GENERAL DE AGUAS-CENTRO DE ESTUDIOS CIENTIFICOS (CECs), 2009. Estrategia Nacional de Glaciares, Fundamentos. 290p.}

FAO, 2012. Diagnóstico Nacional de Montaña, fortalecimiento de la gestión participativa para el desarrollo sostenible de los Andes. Informe Chile. 64p.

INSTITUTO NACINAL DE ESTADÍSTICAS (INE), 2010. Compendio Estadístico, Estadísticas Demográficas. 140p.

MILLENIUM

ECOSYSTEM

ASSESSMENT, 2005. Ecosystems and Human Well-Being: Synthesis. Island Press, Washington, DC. 155p.

MINISTERIO DE AGRICULTURA, 1984. Crea un Sistema Nacional de Áreas Silvestres Protegidas del Estado. 10p. 
MINREL, 1967. Dto-531, Convención para la Protección de la Flora, Fauna y las Bellezas Escénicas Naturales de América, firmado en Washington el 12 de Octubre de 1940. BCN. 5p.

MINSEGPRES, 1994. Ley No19300, Aprueba ley sobre Bases Generales del Medio Ambiente. BCN. 38p.

MINSEGPRES, 2001. D.S. No. 95 de 2001, Reglamento del Sistema de Evaluación de Impacto Ambiental. 47p.

MINSEGPRES, 2010. Ley $\mathrm{N}^{\circ} 20.417$, Crea el Ministerio del Medio Ambiente, el Servicio de Evaluación Ambiental y la Superintendencia del Medio Ambiente. BCN. 55p.
PATERSON, W.S.B., 1994. The Physics of Glaciers. Third Edition. 481p.

PEÑA, H. \& B. NAZARALA, 1987. Snowmelt-runoff simulation model of a central Chile Andean basin with elevant orographic effects. Large Scale effects of Seasonal Snow cover (Proceedings of the Vancouver Symposium, August 1987). IAHSPubl. no. $166.12 \mathrm{p}$.

SEGOVIA, A., 2014. Caracterización Glaciológica de Chile y Valoración de Servicios Ecosistémicos de Glaciares en base a Mercados Reales (Estudio de caso del Monumento Natural el Morado), Tesis de Magíster, Universidad de Chile. Chile,168p. Inédito. 


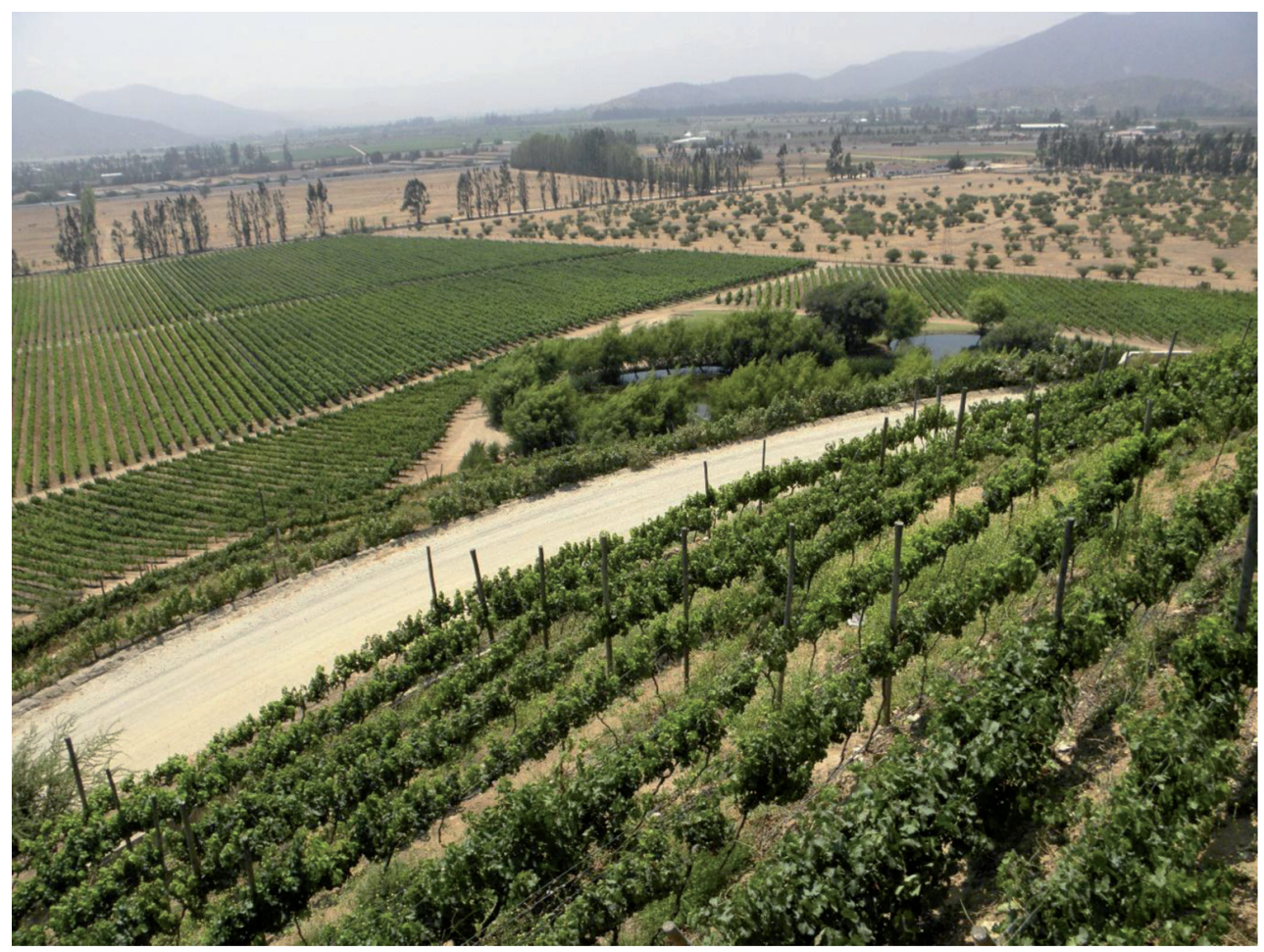

Casablanca Valley, Valparaiso Region, Chile. View toward the valley from a vineyard winery and restaurant. Plantation of Sauvignon Blanc grapes on the slope. This type of grape represents THE LARGEST ACREAGE IN THE VALLEY WITH $80 \%$ OF PRODUCTION DESTINED FOR EXPORT. IPGH / OEA Project. Dr. Fernando Pino, frino@uchilefau.cl 